TRABAJO ORIGINAL

\title{
DIABETES MELLITUS TIPO 1: EDAD DE COMIENZO Y PRESENCIA EN FAMILIARES DE PRIMER GRADO
}

\author{
TYPE 1 DIABETES MELLITUS: AGE OF ONSET \\ AND PRESENCE IN FIRST-DEGREE RELATIVES
}

\begin{abstract}
Edgardo Trinajstic ${ }^{2}$, Alejandra Cicchitti ${ }^{2,3}$, Joaquín González ${ }^{1,2,3}$, Celina Bertona ${ }^{2,3}$, Zelmira Guntsche ${ }^{2,5}$, Patricia Lemos ${ }^{2,4}$, Luciano Ortiz ${ }^{7}$, Eligio Negri ${ }^{2,4}$, Alfredo Bonadé ${ }^{9}$, Roxana Abeledo ${ }^{8}$, Romina Sosa ${ }^{2,10}$, Laura Dimov ${ }^{2,6}$, Gabriela Negri², Martín Rodríguez ${ }^{1,2}$. En representación del Grupo de Estudio MENDODIAB-1
\end{abstract}

\section{RESUMEN}

Introducción: la diabetes mellitus tipo 1 (DM1) se considera una enfermedad de la infancia/adolescencia, pero puede ocurrir a cualquier edad. El riesgo de desarrollar DM1 es de ocho a 15 veces mayor en parientes de primer grado. La proporción de menores de 15 años con un pariente de primer grado afectado es $\approx 12 \%$. Luego de años de seguimiento, esta frecuencia puede aumentar a más del $20 \%$.

Objetivos: determinar la edad de comienzo en pacientes adultos con DM1 y su frecuencia en familiares de primer grado.

Materiales y métodos: en 414 sujetos se definió diagnóstico de DM1 por fenotipo, juicio clínico y requerimiento de insulina dentro del año de diagnóstico. Se determinó edad al diagnóstico y los sujetos se dividieron en mayores y menores de 30 años al momento del debut. En ellos se evaluó y comparó: DM1 en familiares de primer grado, índice de masa corporal (IMC), circunferencia de cintura, HbA1c e hipotiroidismo. Para la comparación de variables cuantitativas de los dos grupos se usó test de $t$ y ANOVA para grupos múltiples, y para las variables categóricas se empleó chi cuadrado con corrección de Fisher.

Resultados: el debut antes de los 10 años fue de $28,2 \%$, entre los 10 y 20 años de $36,9 \%$, entre los 20 y 30 años de $18,8 \%$ y por encima de los 30 años de 17,1\%. Hubo similar frecuencia de hipotiroidismo y familiares de primer grado con DM1 en aquellos con diagnóstico antes o después de los 30 años. Se halló presencia de DM1 en familiares de primer grado en el $17,2 \%$ (padre 3,6\%, madre 3,1\%, hermano 10,2\%, hijo 0,5\%).

Conclusiones: antes de los 30 años debutó el 82,9\% de los pacientes, pero hubo un $17,1 \%$ que lo hizo después. El 17,2\% tenía familiares de primer grado con DM1. A diferencia de los estudios en niños y adolescentes, y al igual que los estudios que evaluron poblaciones de DM1 adultos, encontramos el mayor antecedente de DM1 entre hermanos (10,2\%). Dicho patrón familiar fue similar entre DM1 con comienzo antes o después de los 30 años.

Palabras clave: diabetes mellitus tipo 1; edad de inicio; parientes de primer grado.

Revista de la Sociedad Argentina de Diabetes 2020; Vol. 54 (15-20)

\section{ABSTRACT}

Introduction: type 1 diabetes mellitus (T1DM) is a childhood and adolescence disease, but it can occur at any age. The risk of developing T1DM is 8-15 times higher in first-degree relatives of patients with T1DM. The proportion of affected first-degree relatives in children under 15 with T1DM is $\approx 12 \%$. This frequency may increase to more than $20 \%$ after years of follow-up.

Objectives: to determine the age of onset of T1DM in adult patients and the frequency of T1DM in their first-degree relatives. Materials and methods: a diagnosis of T1DM was defined in 414 subjects by phenotype, clinical judgment and early insulin requirement within the year of diagnosis. Age at diagnosis was determined and subjects were divided into those with debut before or after 30. The following variables were recorded and compared: presence of T1DM in first-degree relatives, body mass index (BMI), waist circumference, HbA1c and association with hypothyroidism. $t$ test and ANOVA for two or multiple subgroups comparison and chi square test with Fisher correction for quantitative or categorical variables were used.

Results: the frequency of diabetes debut before the age of 10 was $28.2 \%$, between 10 and 20 years 36.9\%, between 20 and 30 years $18.8 \%$ and above 30 years $17.1 \%$. The frequency of hypothyroidism and first-degree relatives with T1DM was similar in those diagnosed before or after 30 years. The presence of T1DM in first-degree relatives was found in $17.2 \%$ of the patients (father $3.6 \%$, mother $3.1 \%$, sibling $10.2 \%$, son $0.5 \%$ ).

Conclusions: in $82.9 \%$ of patients debut was before the age of 30 , but it was later in $17.1 \% .17 .2 \%$ of patients had first-degree relatives with T1DM. We found the highest frequency of T1DM association among siblings (10.2\%) similar to studies in adult T1DM populations but contrary to studies in children and adolescents. This family pattern was similar between T1DM beginning before or after 30 years.

Key words: type 1 diabetes mellitus; age of onset; first-degree relatives.

Revista de la Sociedad Argentina de Diabetes 2020; Vol. 54 (15-20) 
1 Instituto de Clínica Médica y Diabetes, staff médico, Mendoza, Argentina

2 Docente, Facultad de Ciencias Médicas, Universidad Nacional de Cuyo, Mendoza, Argentina

3 Médico de Planta, Hospital Universitario, Universidad Nacional de Cuyo, Mendoza, Argentina

4 Médico de Planta, Hospital Central de Mendoza, Mendoza, Argentina

5 Jefa del Servicio de Endocrinología, Hospital Humberto Notti, Mendoza, Argentina

6 Médico de Planta, Hospital José Néstor Lencinas, Mendoza, Argentina

7 Médico, Servicios Médicos Ambulatorios (SEMA-PAMI), Mendoza, Argentina
8 Médico de Planta, Hospital Diego Paroissien, Mendoza, Argentina

9 Médico de Planta, Hospital Italiano, Mendoza, Argentina

10 Médico de Planta, Hospital Del Carmen, Mendoza, Argentina

Contacto del autor: Martín Rodríguez

E-mail: clindiab@gmail.com

Correspondencia: Boulogne Sur Mer 1585, CP5500, Mendoza, Argentina

Fecha de trabajo recibido: 27/01/2020

Fecha de trabajo aceptado: 10/02/20

Conflictos de interés: los autores declaran que no existe conflicto de interés

\section{INTRODUCCIÓN}

La diabetes mellitus tipo 1 (DM1) es causada por la destrucción autoinmune de las células $\beta$ pancreáticas en individuos genéticamente predispuestos y da como resultado una deficiencia severa de insulina. Por lo general, se considera una enfermedad de la infancia y la adolescencia, pero puede ocurrir a cualquier edad. El diagnóstico correcto de DM1 en jóvenes (<20 años) suele ser sencillo porque representa la mayoría de los casos de diabetes en esa población. Por el contrario, la identificación de DM1 en adultos mayores de 30 años es un desafío debido a la prevalencia mucho más alta de DM2 en esa población.

El apoyo diagnóstico con el dosaje de péptido-C y de anticuerpos es de utilidad, aunque no siempre definitorio y con dificultades en nuestro medio. Los autoanticuerpos contra el antígeno decarboxilasa del ácido glutámico (anti GAD) se han utilizado para el diagnóstico, pero sólo están presentes en el 70\% de los pacientes con DM11. La baja incidencia de DM1 en comparación con DM2 en personas mayores de 30 años significa que la proporción de pacientes con DM que tiene una prueba anti GAD positiva verdadera (aproximadamente del 3 al $5 \%$ de todos los casos de DM) es similar a la proporción con una prueba de falsos positivos (alrededor del 2 al 5\%) $^{2}$. Combinando fenotipo y susceptibilidad genética, un reciente estudio de Thomas et al. determinó en europeos que el $42 \%$ de los DM1 tuvo un comienzo luego de los 30 años $^{3}$.

Varios estudios informaron una prevalencia de DM1 en familiares de primer grado dentro de un rango del 5 al 19\%. La proporción de niños con un pariente de primer grado afectado en el momento del diagnóstico es de 10 a $12 \%{ }^{4}$, y después de décadas de seguimiento, esta frecuencia aumenta a más del $20 \%{ }^{5}$. Los padres transmiten la enfermedad a sus hijos con más frecuencia que las madres $^{6}$. En consecuencia, en el momento del diagnóstico, el 4-7\% de los niños tiene un padre con DM1, mientras que sólo 1,5-3\% tienen una madre afectada. Sin embargo, varios de estos estudios no son directamente comparables. En algunos, las poblaciones del estudio comprendían sólo niños o adolescentes como probandos (casos índice) y en familiares de primer grado algunos incluían padres y hermanos, y otros padres, hijos y hermanos. Por ejemplo, el estudio finlandés de Parkkola et al. en niños y adolescentes con DM1 menores de 15 años, encontró DM1 en el 12,2\% de los familiares de primer grado (padres y/o hermanos) ${ }^{4}$, mientras que el estudio danés de Lorenzen et al. en DM1 mayores de 50 años con más de 30 años de diabetes, halló DM1 en el 25,1\% (padres, hijos y/o hermanos) $)^{5}$. Además, se han reportado marcadas diferencias en las tasas de incidencia de DM1 entre grupos raciales y países, y pueden ser en parte responsables de la variación en los resultados?.

\section{OBJETIVOS}

El objetivo del presente estudio fue evaluar la edad de comienzo de DM1 y la presencia de DM1 entre familiares de primer grado (padres, hermanos e hijos) en pacientes con DM1 adultos de la provincia de Mendoza, Argentina.

\section{MATERIALES Y MÉTODOS}

Por interrogatorio, examen físico y registro de historias clínicas, entre junio de 2017 y abril de 2018, se evaluaron sujetos con DM1 $\geq 18$ años, habitantes de 14 departamentos de la provincia de Mendoza, con la participación de 38 médicos con formación diabetológica (Grupo de Estudio MENDODIAB-1).

Los médicos participantes, junto con los pa- 
cientes, previa conformidad verbal, completaron una base de datos que cargaron en forma anónima. Cada médico incorporó de manera consecutiva a pacientes con diagnóstico de DM1. Se definió diagnóstico de DM1 por juicio clínico no estructurado (teniendo en cuenta el índice de masa corporal -IMC-, baja historia familiar de DM2, enfermedades autoinmunes asociadas) y requerimiento de insulina dentro del año de diagnóstico. En ellos se estableció la presencia de DM1 en familiares de primer grado (padres, hermanos e hijos). También se determinó edad al diagnóstico y se dividió a los sujetos en mayores y menores de 30 años al momento del debut, y entre ellos se comparó IMC, circunferencia de cintura, HbA1c y diagnóstico de hipotiroidismo.

\section{Análisis estadístico}

Las variables cualitativas se analizaron por test de chi cuadrado y las cuantitativas por test de ANOVA I corregidas por test de Tukey para comparaciones múltiples. Un valor de $p<0,05$ se consideró estadísticamente significativo. En todos los casos se utilizó un intervalo de confianza del 95\%.

Para tener en cuenta los posibles efectos de confusión sobre la variable de respuesta $\mathrm{HbA} 1 \mathrm{c}$, como edad, duración de la diabetes, edad al inicio de la enfermedad, circunferencia de cintura y presión arterial sistólica, se realizó un análisis de regresión logística con el programa MedCalc.

Según la Encuesta Nacional de Factores de Riesgo (ENFR) de 2013, la prevalencia de diabetes en la provincia de Mendoza fue de 8,9\% en mayores de 18 años. Mendoza tiene un total de 1.886 .000 habitantes y el $80 \%$ de la población es mayor de 20 años, lo cual hace una estimación de $\approx 130.000$ adultos con diabetes. Si del 5 al 10\% corresponde a DM1, podría considerarse una cifra entre $\approx 6.500$ y 13.000 adultos con DM1. Para un universo con ese número de DM1 se definió el tamaño muestral a través del sample size calculator MedCalc en 363 a 374 sujetos, con un margen de error del $5 \%$ y un nivel de confianza del $95 \%$.

\section{RESULTADOS}

Se incluyeron 414 pacientes con DM1 $\geq 18$ años, mujeres $52 \%$, edad $34,8 \pm 13,9$ años. El debut por edad se muestra en la Figura 1: antes de los 10 años $28,2 \%$, entre los 11 y 20 años 36,9\%, entre los 21 y 30 años 18,8\% y 17,1\% luego de los 30 años. Las mujeres debutaron con mayor frecuencia que los varones antes de los 10 años $(37,0 \%$ vs $18,6 \% ; p<0,001)$ y más los varones entre los 11 y 20 años $(42,7 \%$ vs $31,5 \% ; p=0,017)$. No hubo diferencias después de los 30 años (varones $19,1 \%$ y mujeres $15,3 \% ; p=0,3)$. La comparación entre $\geq 30$ años y $<30$ años al debut se muestra en la Tabla 1. Entre ambos grupos no hubo diferencias en la concomitancia de hipotiroidismo, control metabólico ni en la frecuencia de familiares de primer grado con DM1. Quienes debutaron con más de 30 años eran mayores $(50,2$

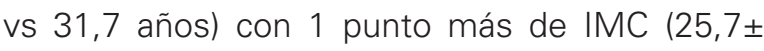
vs $\left.24,7 \pm \mathrm{kg} / \mathrm{m}^{2}\right)$, mayor circunferencia de cintura $(88,1 \pm 11,9$ vs $84,4 \pm 13,6 \mathrm{~cm})$ y menor antigüedad de DM (13 $\pm 9,8$ años vs $17,4 \pm 12,3$ años).

El $82,8 \%$ de DM1 fue de aparición esporádica. La frecuencia de familiares de primer grado (padre, madre, hermanos o hijos) con DM1 fue del $17,2 \%$. La mayor frecuencia fue en hermanos $(10,2 \%)$, luego padre $(3,6 \%)$, madre $(3,1 \%)$ e hijos $(0,5 \%)$. Dicho patrón familiar fue similar entre DM1 con comienzo antes o después de los 30 años (Figura 2).

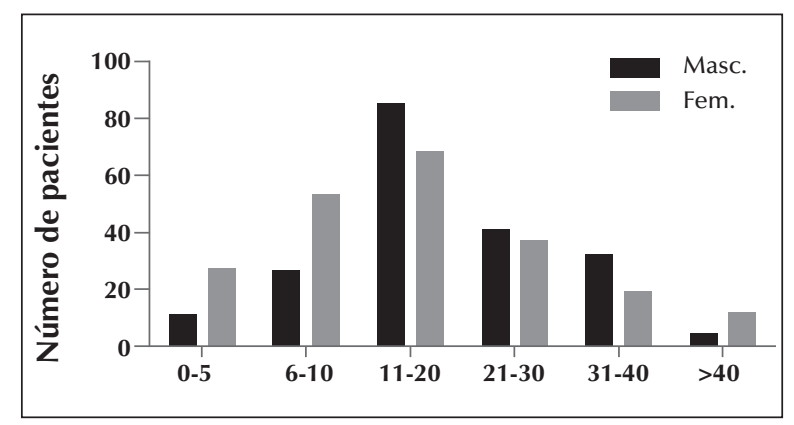

Figura 1: Edad al debut de DM1. 


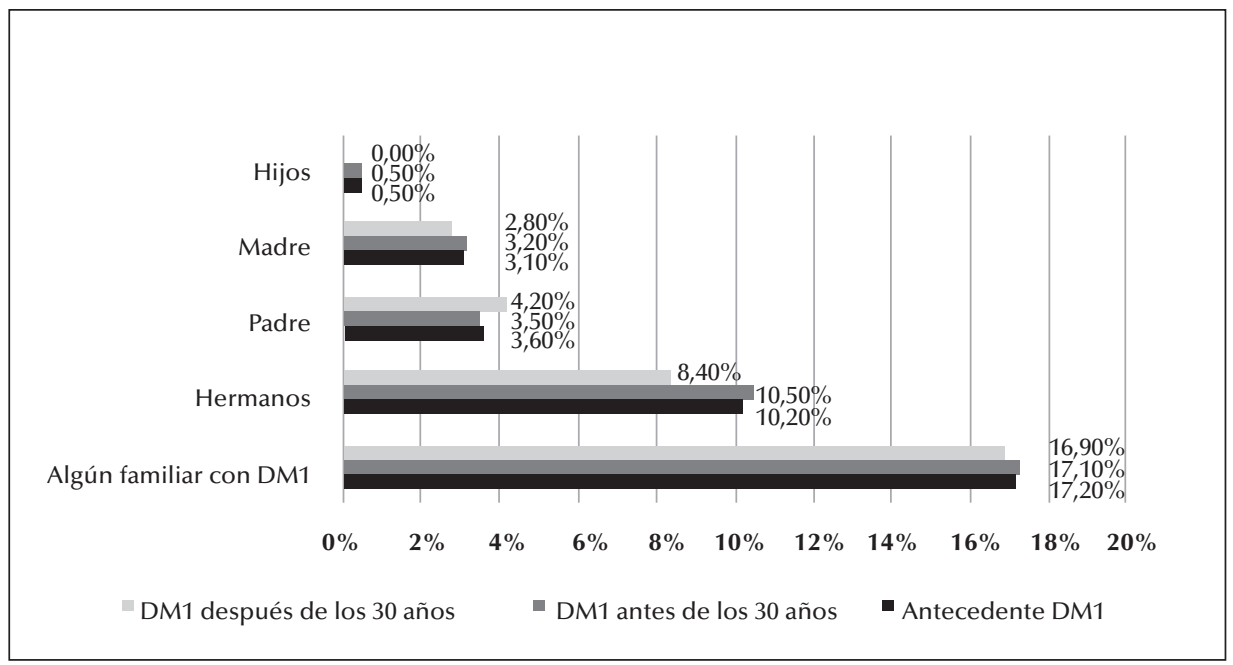

Figura 2: Antecedente de DM1 en padre, madre, hermanos o hijos en pacientes con DM1, global y según debut antes o después de los 30 años.

\begin{tabular}{|l|l|l|l|}
\hline & \multicolumn{1}{|c|}{ Diagnóstico $<\mathbf{3 0}$ años } & \multicolumn{1}{c|}{ Diagnóstico $\geq \mathbf{3 0}$ años } & \\
\hline $\mathrm{N}$ total 414 & $344(82,9 \%)$ & $71(17,1 \%)$ & \\
\hline Varones 199 & $80,9 \%$ & $19,1 \%$ & 0,2402 \\
\hline Mujeres 216 & $84,7 \%$ & $15,3 \%$ & 0,2402 \\
\hline Edad al diagnóstico (años) & $14,3 \pm 7$ & $37,2 \pm 6,3$ & $<0,0001$ \\
\hline Edad actual & $31,7 \pm 12$ & $50,2 \pm 12,5$ & $<0,0001$ \\
\hline Familiares de primer grado con DM1 & $17,1 \%$ & $16,9 \%$ & 1,0 \\
\hline IMC & $27,7 \pm 4,2$ & $25,7 \pm 3,6$ & 0,031 \\
\hline Circunferencia de cintura (cm) & $84,4 \pm 13,6$ & $88,1 \pm 11,9$ & 0,0169 \\
\hline HbA1c<7\% & $20,2 \%$ & $20,5 \%$ & 0,11 \\
\hline $7-7,9 \%$ & $29,1 \%$ & $41,1 \%$ & 0,11 \\
\hline$\geq 8$ & $50,6 \%$ & $38,2 \%$ & 0,11 \\
\hline Hipotiroidismo + & $25 \%$ & $29,5 \%$ & 0,45 \\
\hline Antigüedad (años) & $17,4 \pm 12,3$ & $13 \pm 9,8$ & 0,0253 \\
\hline Mediana (SDs) & 16,62 & 12,84 & 16,02 \\
\hline Mediana (SDs) & 16,62 & 12,84 & 16,02 \\
\hline Mediana (SDs) & 16,62 & 16,62 & 16,62 \\
\hline
\end{tabular}

Tabla 1: Comparación entre pacientes DM1 con debut antes o después de los 30 años.

\section{DISCUSIÓN}

Sobre una población total de 502.667 individuos, Thomas et al. encontraron 13.250 pacientes diagnosticados con DM hasta los 60 años. Con el uso de un score de alta susceptibilidad genética (usando variantes asociadas con riesgo de DM1), la prevalencia de DM1 en esa población fue de 9,7\% (1.286 pacientes), con $58 \%$ de los casos diagnosticados en menores de 30 años y un $42 \%$ entre 31 y 60 años. En dicho trabajo el fenotipo fue similar en aquellos diagnosticados antes o después de los 30 años con similar IMC al ingreso al estudio (con diagnóstico $<30$ años $26,7[26,4-27,0] \mathrm{kg} / \mathrm{m}^{2}$ y con diagnóstico $\geq 30$ años $\left.27,4[26,7-28,0] \mathrm{kg} / \mathrm{m}^{2}\right)$, rápido progreso a la necesidad de insulina y similar debut con cetoacidosis $(9 \%$ vs $11 \%)$. El requerimiento de insulina dentro del año de diagnóstico fue del $97 \%$ en los diagnosticados antes de los 30 años y del 89\% en los diagnosticados después de los 30 años ${ }^{3}$.

En nuestro trabajo reunimos información de 414 pacientes adultos con DM1. El diagnóstico de DM1 se estableció sobre la base del juicio clíni- 
co y el requerimiento de insulina dentro del año de diagnóstico, sin establecer restricciones respecto de la edad de comienzo. Hallamos que el $17,1 \%$ de los pacientes con DM1 fue diagnosticado luego de los 30 años. También el IMC actual fue similar en ambos grupos (con diagnóstico $<30$ años $24,7 \pm 4,2 \mathrm{~kg} / \mathrm{m}^{2}$ y con diagnóstico $\geq 30$ años $\left.25,7 \pm 3,6 \mathrm{~kg} / \mathrm{m}^{2}\right)$. No hubo diferencia en la frecuencia de DM1 en familiares de primer grado (con diagnóstico $<30$ años $17,1 \%$ y con diagnóstico $\geq 30$ años $16,9 \% ; p=1,0$ ) y tampoco en la frecuencia de hipotiroidismo asociado (con diagnóstico $<30$ años $25 \%$ y con diagnóstico $\geq 30$ años $29,5 \% ; p=0,45$ ), lo que hace pensar en una adecuada aproximación diagnóstica.

En niños y adolescentes del registro nacional de Finlandia, Parkkola et al. encontraron que un 12,2\% tenía un familiar de primer grado (padres o hermanos) con DM1 (padre 6,2\%, madre 3,2\% y hermano $2,9 \%)^{4}$. Cuando el análisis se hace a partir de probandos de mayor edad, el porcentaje aumenta. Por ejemplo, Lorenzen y Nerup, en 1994 evaluaron la presencia de familiares de primer grado con DM1 en 291 probandos mayores de 50 años y encontraron un porcentaje mayor: 25,1\% (padre 3,8\%, madre $2,4 \%$ hijos $6,5 \%$, hermano $16,5 \%$ ) y debe considerarse que el $37 \%$ de los probandos no tenía hermanos ni hijos y fueron incluidos como "esporádicos". No se observaron diferencias estadísticamente significativas en los riesgos para los familiares de primer grado de probandos con edad de inicio antes y después de los 20 años. Y también encontraron, de acuerdo con informes anteriores, que la edad de inicio en los casos índice (probandos) fue significativamente menor que en los segundos casos. En el $48 \%$ de los pares de hermanos concordantes para DM1, el segundo caso se diagnosticó más de 10 años después del caso índice ${ }^{8}$.

Nuestra muestra incluyó pacientes con una edad de 34,8 $\pm 13,9$ años, varios de ellos sin hijos y con hermanos menores aún sin DM1. Determinamos la presencia de DM1 en familiares de primer grado en el 17,2\% (padre 3,6\%, madre 3,1\%, hermano $10,2 \%$, hijo 0,5\%).

Es evidente que cuando mayor es la edad de los probandos, la frecuencia familiar de DM1 es mayor sobre todo a expensas de un aumento en hermanos e hijos. Se considera que el riesgo de desarrollar DM1 es ocho a 15 veces mayor en familiares de primer grado 9 . Considerando las mejores condiciones para que las personas con DM1 puedan lograr tener uno o más hijos, es probable que el antecedente de familiares con DM1 sea cada vez mayor.

Nuestro trabajo presenta debilidades. El diagnóstico de DM1 fue sólo por juicio clínico del médico tratante y comienzo de insulinoterapia dentro del año de diagnóstico y no disponemos de la confirmación inmunológica, de péptido $\mathrm{C}$ o genética para reafirmar el diagnóstico, principalmente en mayores de 30 años. Los datos de familiares con DM1 fueron referidos por los propios pacientes y no siempre pudieron confirmarse.

\section{CONCLUSIONES}

Antes de los 30 años debutó el $82,9 \%$ de los pacientes, pero hubo un $17,1 \%$ que lo hizo después. Igual asociación con hipotiroidismo y familiares de primer grado con DM1 sugiere una correcta aproximación diagnóstica en mayores de 30 años sobre la base del fenotipo y criterio clínico. El 17,2\% tenía familiares de primer grado con DM1. A diferencia de los estudios en niños y adolescentes, y coincidiendo con aquellos que evaluaron poblaciones de DM1 adultos, encontramos el mayor antecedente de DM1 entre hermanos (10,2\%). Dicho patrón familiar fue similar entre DM1 con comienzo antes o después de los 30 años.

El presente trabajo fue presentado en el XXI Congreso Argentino de Diabetes 2018 en dos pósteres: póster No 90 "Antecedente de diabetes mellitus tipo 1 (DM1) en familiares de primer grado en adultos DM1"; Póster No 91 "Edad de comienzo de diabetes mellitus tipo 1."

\section{Miembros del Grupo de Estudio de Diabetes Tipo 1 de Mendoza (MENDODIAB-1)}

Martín Rodríguez, Edgardo Trinajstic, Alejandra Cicchitti, Joaquín González, Celina Bertona, Víctor Previtera, Nelson Rodríguez, Pedro Calella, Pablo Ávila, Soledad Acosta, María Inés Argerich, Carolina Dromi, Patricia Lemos, Franco Bellomo, Laura Bidot, Karina Mengoni, Daniel Giorgini, Liliana Abdala, Gabriela Negri, Carla Ponce, Luciano Ortiz, Eligio Negri, Macarena Argumedo, Gabriel Minuchín, Laura Dimov, Luis Lombardo, Paula Muñoz, Romina Sosa, Zelmira Guntsche, Roxana Abeledo, Laura Cuello, Alfredo Bonadé, Norma Carrasco, Leticia Barrera, Raúl David, Laura Romero, Luis Biliato, Hugo Lavandaio. 


\section{BIBLIOGRAFÍA}

1. Sabbah E, Savola K, Ebeling T, et al. Genetic, autoimmune, and clinical characteristics of childhood and adultonset type 1 diabetes. Diabetes Care 2000; 23: 1326-32.

2. Bingley PJ. Clinical applications of diabetes antibody testing. J Clin Endocrinol Metab 2010; 95: 25-33.

3. Thomas NJ, Jones SE, Weedon MN, et al. Frequency and phenotype of type 1 diabetes in the first six decades of life: a cross-sectional, genetically stratified survival analysis from UK Biobank. Lancet Diabetes Endocrinol 2018; 6: 122-129.

4. Parkkola A, Härkönen T, Ryhänen $S$, et al. Extended family history of yype 1 diabetes and phenotype and genotype of newly diagnosed children. Diabetes Care 2013 Feb; 36: 348-54.
5. Lorenzen T, Pociot F, Nerup J, et al. Long-term risk of IDDM in first-degree relatives of patients with IDDM. Diabetologia 1994; 37:321-327.

6. Harjutsalo V, Reunanen A, Tuomilehto J. Differential transmission of type 1 diabetes from diabetic fathers and mothers to their offspring. Diabetes 2006; 55: 1517-1524.

7. Spanakis E, Golden SH. Race/ethnic difference in diabetes and diabetic complications. Curr Diab Rep 2013; 13: 814-823.

8. Wagener DK, Sacks J, LaPorte R, et al. The Pittsburg Study of insulin-dependent diabetes mellitus. Risk for diabetes among relatives of IDDM. Diabetes 1982; 31: 136-144.

9. Sipetic S, Vlajinac $\mathrm{H}$, Kocev $\mathrm{N}$, et al. Family history and risk of type 1 diabetes mellitus. Acta Diabetol 2002; 39: 111-115. 